\title{
Childhood cartilage ECM enhances the chondrogenesis of endogenous cells and subchondral bone repair of the unidirectional collagen-dECM scaffolds in combination with microfracture
}

Hongfu Cao ${ }^{1,2}$, Xiuyu Wang ${ }^{3}$, Manyu Chen ${ }^{1,2}$, Yuhan Liu ${ }^{3}$, Xiaolin Cui ${ }^{4,5}$, Jie Liang ${ }^{1,2}$, Qiguang Wang ${ }^{1,2 *}$, Yujiang Fan ${ }^{1,2}$, and Xingdong Zhang ${ }^{1,2}$

1. National Engineering Research Center for Biomaterials, Sichuan University, 29 Wangjiang Road, Chengdu, Sichuan, 610065, China

2. College of Biomedical Engineering, Sichuan University, 29 Wangjiang Road, Chengdu, Sichuan, 610065, China

3. Guangxi Engineering Center in Biomedical Materials for Tissue and Organ Regeneration, Guangxi Collaborative Innovation Center for Biomedicine, Guangxi Medical University, Nanning, Guangxi, 530021, China

4. Department of Orthopaedic Surgery, University of Otago, Christchurch, 8011, New Zealand

5. Department of Bone and Joint, The First Affiliated Hospital of Dalian Medical University, Dalian, Liaoning, 116000, China

\section{Corresponding Author}

*Email: wqgwang@126.com 
Table. S1. Sequence of related gene primers

\begin{tabular}{ccc}
\hline Gene & Forward primer & Reverse primer \\
\hline GAPDH & TCGGAGTGAACGGATTTGGC & TTCCCGTTCTCAGCCTTGAC \\
AGG & GGCCACTGTTACCGTCACTT & GTCCTGAGCGTTGTTGTTGAC \\
SOX 9 & TCTGGAGACTGCTGAACGAG & CTGCCCATTCTTCACCGACTT \\
COL II & TGATAAGGATGTGTGGAAGCCG & CAGGCAGTCCTTGGTGTCTTC \\
COL $X$ & TCCCAGA ACCCAGAATCCATC & GGTTGTGGGCCT TTTATGCC \\
\hline
\end{tabular}


Table S2. International Cartilage Repair Society (ICRS) macroscopic evaluation tool

\begin{tabular}{lc}
\hline Categories & Score \\
\hline Degree of defect repair & \\
In level with surrounding cartilage & 4 \\
$75 \%$ repair of defect depth & 3 \\
$50 \%$ repair of defect depth & 2 \\
$25 \%$ repair of defect depth & 1 \\
$0 \%$ repair of defect depth & 0 \\
\hline
\end{tabular}

\section{Integration to border zone}

Complete integration with surrounding cartilage $\quad 4$

Demarcating border $<1 \mathrm{~mm} \quad 3$

3/4th of graft integrated, 1/4th with a notable border $>1 \mathrm{~mm}$ width 2

$1 / 2$ of graft integrated with surrounding cartilage, $1 / 2$ with a notable border $>1 \mathrm{~mm} \quad 1$

From no contact to $1 / 4$ th of graft integrated with surrounding cartilage $\quad 0$

\section{Macroscopic appearance}

$\begin{array}{ll}\text { Intact smooth surface } & 4\end{array}$

$\begin{array}{ll}\text { Fibrillated surface } & 3\end{array}$

\begin{tabular}{l|l} 
Small, scattered fissures or cracks & 2
\end{tabular}

Several, small or few but large fissures $\quad 1$

$\begin{array}{ll}\text { Total degeneration of grafted area } & 0\end{array}$

\section{Overall repair assessment}

Grade I: normal 12

Grade II: nearly normal $\quad$ 8-11

Grade III: abnormal

Grade IV: severely abnormal $\quad 1-3$ 
Table S3. Histological scoring system

\begin{tabular}{|c|c|}
\hline Category & Points \\
\hline \multicolumn{2}{|l|}{ Cell morphology } \\
\hline Hyaline cartilage & 4 \\
\hline Mostly hyaline cartilage & 3 \\
\hline Mostly fibrocartilage & 2 \\
\hline Mostly non-cartilage & 1 \\
\hline Non-cartilage only & 0 \\
\hline \multicolumn{2}{|l|}{ Matrix staining } \\
\hline Normal & 3 \\
\hline Slightly reduced & 2 \\
\hline Markedly reduced & 1 \\
\hline No metachromatic stain & 0 \\
\hline \multicolumn{2}{|l|}{ Surface regularity } \\
\hline Smooth $(>3 / 4)$ & 3 \\
\hline Moderate $(>1 / 2-3 / 4)$ & 2 \\
\hline Irregular $(1 / 4-1 / 2)$ & 1 \\
\hline Severely irregular $(<1 / 4)$ & 0 \\
\hline \multicolumn{2}{|c|}{ Integration of donor with host adjacent cartilage } \\
\hline Both edges integrated & 2 \\
\hline One edge integrated & 1 \\
\hline Neither edge integrated & 0 \\
\hline
\end{tabular}

